\title{
NEW ASPECTS OF SUBFITNESS \\ IN FRAMES AND SPACES
}

\author{
JORGE PICADO AND ALEŠ PULTR \\ In memory of Horst Herrlich
}

\begin{abstract}
This paper contains some new facts about subfitness and weak subfitness. In the case of spaces, subfitness is compared with the axiom of symmetry, and certain seeming discrepancies are explained. Further, Isbell's spatiality theorem in fact concerns a stronger form of spatiality ( $T_{1}$-spatiality) which is compared with the $T_{D}$-spatiality. Then, a frame is shown to be subfit iff it contains no non-trivial replete sublocale, and the relation of repleteness and subfitness is also discussed in spaces. Another necessary and sufficient condition for subfitness presented is the validity of the meet formula for the Heyting operation, which was so far known only under much stronger conditions.
\end{abstract}

\section{INTRODUCTION}

Subfitness is a low separation axiom, in the context of spaces weaker than $T_{1}$. It was introduced by Isbell in his pioneering article [8], but almost immediately dismissed for its bad categorical behavior. The only merit mentioned was its role in the spatiality of compact frames; the attention was concentrated on the stronger fitness, which might have seemed not a very radical modification (being in fact a hereditary subfitness), and the categorical properties of which were excellent. Later, subfitness appeared sporadically in literature (with both a topological and logical motivation in [16], as a necessary and sufficient condition for admitting a generalized nearness in [7] - coauthored by Horst Herrlich -, and in a few other papers). In [17], it was indicated that the condition is by no means uninteresting, and quite recently ([14]) the authors of the present

Date: May 19, 2016.

2010 Mathematics Subject Classification. 06D22, 54B05, 54D10, 54E15, 54E17.

Key words and phrases. frame (locale), sublocale, sublocale lattice, subfitness, $T_{1}$ spatiality, $T_{D}$-spatiality, repleteness, nearness.

Support from the project P202/12/G061 of the Grant Agency of the Czech Republic and from the Centre for Mathematics of the University of Coimbra (funded by the European Regional Development Fund program COMPETE and by the project PEstC/MAT/UI0324/2013 of the Fundação para a Ciência e a Tecnologia) is gratefully acknowledged. 
article analyzed (a.o.) the role of subfitness as a supportive property in combination with other conditions, and its relation with the fitness. The latter did not bring very good news: fitness turned out to be actually a very strong property akin to regularity so that what might have seemed a harmless modification of a (very much needed) low separation axiom for categorical purposes turned out to be a rather strong one. Thus, it seems that the properties of the non-amended subfitness should be given more attention.

This paper is concerned with some of such properties, that is, properties of subfitness as such, not adapted or extended by other conditions.

After necessary Preliminaries in the short Section 2 we study the subfitness in the context of spaces. Here, this property makes perfectly good sense, but there is a seeming discrepancy with the axiom of symmetry, which is explained. In Section 3 we show that Isbell's spatiality theorem in fact says more, namely that the space in question is $T_{1}$-spatial (that is, isomorphic to $\Omega(X)$ with a $T_{1}$-space $\left.X\right) . T_{1}$-spatiality is then treated in more generality, compared with $T_{D}$-spatiality and it is proved (a.o.) that a $T_{D}$-spatial frame is $T_{1}$-spatial iff it is subfit. The next Section 4 is devoted to repleteness, a property of a space akin to density (see 4.1). We prove that a frame is subfit iff it has no nontrivial replete sublocale. Repleteness may play a more important role in the point-free context than in spaces; but there are some spatial questions calling for explanation which is provided in the second part of this section. Finally, in Section 5 we show that subfitness is equivalent with the validity of the meet formula for the Heyting operation $a \rightarrow b=\bigwedge\{x \mid a \vee x=1, x \geq b\}$ (and that weak subfitness - a weaker condition, see 1.4.1 - is equivalent with the validity of the meet formula for the pseudocomplement $\left.a^{*}=\bigwedge\{x \mid a \vee x=1\}\right)$ and add a few comments about this phenomenon.

\section{Preliminaries}

1.1. A frame resp. co-frame is a complete lattice $L$ satisfying

$$
a \wedge(\bigvee B)=\bigvee\{a \wedge b \mid b \in B\} \quad \text { resp. } \quad a \vee(\bigwedge B)=\bigwedge\{a \vee b \mid b \in B\}
$$

for all $a \in L$ and $B \subseteq L$. Thus, in a frame $L$ the mappings $(x \mapsto x \wedge b): L \rightarrow L$ preserve suprema and hence we have the right Galois adjoints $(x \mapsto(b \rightarrow x))$ : $L \rightarrow L$, satisfying

$$
a \wedge b \leq c \quad \text { iff } \quad a \leq b \rightarrow c
$$

and making $L$ a Heyting algebra. The pseudocomplement (that is, $a \rightarrow 0$ ) will be denoted by $a^{*}$.

A typical frame is the lattice $\Omega(X)$ of all open sets of a topological space $X$. A frame homomorphism $h: L \rightarrow M$ preserves all joins and all finite meets; if 
$f: X \rightarrow Y$ is a continuous map we have a frame homomorphism $\Omega(f): \Omega(Y) \rightarrow$ $\Omega(X)$ defined by $\Omega(f)(U)=f^{-1}[U]$.

1.1.1. The $\Omega$ above is a contravariant functor from the category Top of topological spaces into the category of frames, Frm. It becomes covariant if we consider the opposite category $\mathbf{L o c}=\mathbf{F r m}^{\text {op }}$. It is advantageous to treat the category Loc as a concrete one with the opposite arrows to frame homomorphisms $h: L \rightarrow M$ represented as their right Galois adjoints $f: M \rightarrow$ $L$; these will be referred to as localic maps. Emphasizing this point of view we often speak of frames as of locales.

1.2. $\Omega:$ Top $\rightarrow$ Loc has a right adjoint $\Sigma:$ Loc $\rightarrow$ Top called the spectrum. We will use the description of $\Sigma L$ as the set $\{p \in L \mid p$ prime $\}$ endowed with the topology $\left\{\Sigma_{a} \mid a \in L\right\}$ where $\Sigma_{a}=\{p \mid a \not \leq p\}$, and $(\Sigma f)(p)=f(p)$.

1.3. One thinks of a frame $L$ as of a generalized space. From the several representations of a (generalized) subspace of $L$ we will use that of a sublocale. It is a subset $S \subseteq L$ such that

(S1) $M \subseteq S \Rightarrow \bigwedge M \in S$, and

(S2) $x \in L, s \in S \Rightarrow x \rightarrow s \in S$.

$S$ is a frame in the order of $L$ and inherits its Heyting structure. The embedding $j_{S}: S \subseteq L$ is a localic map (recall 1.1.1: in view of the category Loc, the sublocales in this form are the natural subobjects, that is, "sub-locales"). The corresponding frame homomorphism (the left adjoint)

$$
\nu_{S}: L \rightarrow S
$$

is given by $\nu_{S}(x)=\bigwedge\{s \in S \mid s \geq x\}$. The system of all sublocales constitutes a co-frame

$$
\mathcal{S}(L)
$$

the meets coinciding with the intersections, and the joins defined by

$$
\bigvee S_{i}=\left\{\bigwedge M \mid M \subseteq \bigcup S_{i}\right\}
$$

The top of $\mathcal{S}(L)$ is $L$ and the bottom is the set $\mathrm{O}=\{1\}$; the latter, representing the void subspace, will be referred to as the empty sublocale.

Another representation of sublocales we will sometimes use is that by frame congruences $E_{S}=\left\{(a, b) \in L \times L \mid \nu_{S}(a)=\nu_{S}(b)\right\}$.

1.3.1. Open resp. closed subspaces are represented by open resp. closed sublocales

$$
\mathfrak{o}(a)=\{x \mid a \rightarrow x=x\}=\{a \rightarrow x \mid x \in L\} \text { resp. } \mathfrak{c}(a)=\uparrow a=\{x \mid x \geq a\} .
$$

$\mathfrak{o}(a)$ and $\mathfrak{c}(a)$ are complements of each other. We have (see e.g. [12]):

- $\mathfrak{o}(0)=\mathrm{O}, \mathfrak{o}(1)=L, \mathfrak{o}(a \wedge b)=\mathfrak{o}(a) \cap \mathfrak{o}(b), \mathfrak{o}\left(\bigvee a_{i}\right)=\bigvee \mathfrak{o}\left(a_{i}\right)$, 
- $\mathfrak{c}(0)=L, \mathfrak{c}(1)=\mathrm{O}, \mathfrak{c}(a \wedge b)=\mathfrak{c}(a) \vee \mathfrak{c}(b), \mathfrak{c}\left(\bigvee a_{i}\right)=\bigcap \mathfrak{c}\left(a_{i}\right)$,

- $\mathfrak{o}(a) \cap \mathfrak{c}(b) \neq \mathrm{O}$ iff $a \not \leq b$, and $\mathfrak{c}(a) \subseteq \mathfrak{o}(b)$ iff $a \vee b=1$.

1.3.2. Just like in spaces and subspaces,

open sublocales in a sublocale $S$ are the $\mathfrak{o}_{S}\left(\nu_{S}(a)\right)=S \cap \mathfrak{o}(a)$, and similarly we have the closed sublocales of $S, \mathfrak{c}_{S}\left(\nu_{S}(a)\right)=S \cap \mathfrak{c}(a)$.

One has an extremely simple formula for the closure of $S, \bar{S}=\uparrow(\bigwedge S)$.

1.3.3. Observation. $\mathfrak{o}(a) \cap S \neq \mathrm{O}$ iff $\mathfrak{o}(a) \cap \bar{S} \neq \mathrm{O}$ (since $\mathfrak{o}(a) \cap S=\mathrm{O}$ iff $S \subseteq \uparrow a$ iff $\bar{S} \subseteq \uparrow a)$

1.3.4. An important property of a complemented $S$ is that for any system $T_{i}$, $i \in I$, of sublocales one has

$$
S \cap \bigvee T_{i}=\bigvee\left(S \cap T_{i}\right)
$$

(note that this is exceptional: $\mathcal{S}(L)$ is a co-frame, not a frame; in fact this law characterizes complementarity — see [12, VI.4.4.3]).

1.4. A frame $L$ is subfit if

$$
\forall a, b \in L, \quad a \not \leq b \Rightarrow \exists c, a \vee c=1 \neq b \vee c .
$$

Equivalently, $L$ is subfit iff each open sublocale in $L$ is a join of closed ones.

In fact, this is the original definition, the first order formula above came later (see e.g. [16]).

1.4.1. Of some interest is a weaker property, the weak subfitness (see e.g. [7])

$$
\forall a>0 \exists c \neq 1, a \vee c=1 \text {. }
$$

(weakly subfit)

Subfitness is not a hereditary property, but (see e.g. [12])

1.4.2. A complemented sublocale of a subfit frame is subfit.

For more about frames see e.g. $[9,12,15]$.

\section{LOW SEPARATION AXIOMS: SUbFitNess in SPACES}

2.1. A space $X$ is (of course) subfit if for open $U, V, U \nsubseteq V$ there is an open $W$ such that $U \cup W=X \neq V \cup W$. This property has no relation to $T_{0}$ but

it is obviously implied by $T_{1}$ :

if $x \in U \backslash V$ take $W=X \backslash\{x\}$. It is easy to see that subfitness is strictly weaker than $T_{1}$.

2.1.1. Note. In the standard hierarchy of separation axioms we usually add $T_{1}$ to $T_{3}$ to have an axiom stronger than $T_{2}$. In fact it suffices to add $T_{0}$, that 
is, a regular $T_{0}$-space is Hausdorff. On the other hand, $T_{0}$ does not suffice to make a normal space regular. We have, however,

$$
\text { (normal) \& (subfit) } \Rightarrow \quad \text { (completely regular). }
$$

2.2. Recall that a space $X$ is $T_{D}$ ([1]) if for each $x \in X$ there is an open $U \ni x$ such that $U \backslash\{x\}$ is still open. Obviously $T_{D}$ is strictly stronger than $T_{0}$ and strictly weaker than $T_{1}$. We have

Fact. $T_{1} \equiv T_{D} \&$ (subfit).

$(\Rightarrow$ is obvious. $\Leftarrow$ : For $x \in X$ choose an open $U \ni x$ such that $V=U \backslash\{x\}$ is open and then an open $W$ such that $U \cup W=W \neq V \cup W$. Then $\{x\}=$ $X \backslash(V \cup W)$ is closed.)

2.3. In spaces, the subfitness is characterized by the following property ([8, $16])$.

Theorem. A space is subfit if and only if for each $x \in X$ and each open $U \ni x$ there is a $y \in \overline{\{x\}}$ with $\overline{\{y\}} \subseteq U$.

This relates subfitness to another weak separation axiom, namely the symmetry, which appeared already in 1951 ([11]) under the somewhat surprising name of weak regularity,

$$
x \in \overline{\{y\}} \Leftrightarrow y \in \overline{\{x\}} . \quad \text { (symmetric) }
$$

Thus, (symmetric) $\Rightarrow$ (subfit), and subfitness is strictly weaker than symmetry. (See the following example: take $\mathbb{N}=\{0,1,2, \ldots\}$ and declare a non-void $U \subseteq \mathbb{N}$ open if $0 \in U$ and $\mathbb{N} \backslash U$ is finite. The resulting space is subfit but not symmetric.)

2.4. However, one immediately sees that a space is symmetric iff each open set is a union of closed ones. It seems to contrast with the characteristics (original definition) of subfitness as in 1.4 and the example above. This calls for an explanation.

The point is in an imperfect representation of subspaces as sublocales in non- $T_{D}$ spaces. A subspace $Y$ of a space $X$ is naturally represented by the sublocale $S_{Y}$ associated with the congruence $E_{Y}$ defined by setting

$$
E_{Y}=\{(U, V) \mid U, V \in \Omega(X), U \cap Y=V \cap Y\} .
$$

The following holds (see [3]):

Theorem. The correspondence $Y \mapsto E_{Y}$ is one-to-one if and only if $X$ is a $T_{D}$-space.

We have already observed that $T_{D} \&$ (subfit) $=T_{1}$. It is equally easy to see that $T_{D} \&$ (symmetric) $=T_{1}$. Hence, in $T_{D}$-spaces (subfit), (symmetric) and $T_{1}$ coincide while in non- $T_{D}$-spaces these properties differ. In the latter 
case, the representation of subspaces (subsets) by sublocales is not perfect, and statements about them have to be interpreted as statements about equivalence classes in the following sense.

Let us say that subsets $Y, Z$ are congruence-equivalent (briefly, cong-equivalent) and write $Y \approx Z$ if $E_{Y}=E_{Z}$. More explicitly

$$
Y \approx Z \quad \equiv_{\text {def }} \quad(\forall \text { open } U, V, \quad U \cap Y=V \cap Y \text { iff } U \cap Z=V \cap Z) .
$$

We will have more about the equivalence $Y \approx Z$ in the section on repleteness below. Now we will only explain the seeming discrepancy between symmetry and subfitness: for non- $T_{D}$ spaces, subfitness in terms of subspaces amounts to each open sets being just cong-equivalent to a join of closed ones.

2.4.1. Note. This also explains the fact that a space $X$ admits a nearness iff it is symmetric (see Herrlich's paper [6]) while a frame $L$ admits a (generalized) nearness iff it is subfit ([7], see also [13]).

\section{Isbell's Spatiality Theorem and $T_{1}$-Spatiality}

3.1. In his pioneering article [8], Isbell proved a simple but important spatiality theorem based on subfitness (and stated that there is probably not much other merit in the concept - which, however, turned out to be a much too pessimistic expectation). Using the first order formulation of subfitness (1.4) this theorem has an extremely short proof (see 3.3 and 3.3 .1 below), and we see that in fact it states more than the plain spatiality.

Stating that $L$ is spatial we claim that it is isomorphic to an $\Omega(X)$ but in general we cannot be sure that the $X$ in question has this or other property. For instance, the $L$ where the $X$ can be chosen to be $T_{D}$ (the $T_{D}$-spatial frames) have special properties which were studied in [4]. Now the Isbell's spatiality theorem guarantees a $T_{1}$-space $X$. This, and the relation to the $T_{D}$-spatiality will be the main topic of this section.

3.2. A frame $L$ is max-bounded if for each $x \in L$ there is a maximal $p \in L$ such that $x \leq p$ (note that each maximal element $p$ is prime, hence such $p$ 's are in $\Sigma L)$. We say that $L$ is $T_{1}$-spatial if for each $x \in L$,

$$
x=\bigwedge\{p \mid p \text { maximal, } x \leq p\} .
$$

Thus, if $L$ is $T_{1}$-spatial then it can be represented as $\Omega\left(\Sigma_{\max }(L)\right)$ where $\Sigma_{\max }(L)$ is the subspace of $\Sigma(L)$ carried by all the maximal $p \in L$.

3.2.1. Note. The space $\Sigma_{\max }(L)$ is $T_{1}$ so that a $T_{1}$-spatial frame is representable by a $T_{1}$-space. The reverse fact, namely that the representability of as $\Omega(X)$ with a $T_{1}$-space $X$ implies ( $T_{1}$-spatial), is not quite so obvious. But see 3.6.1 below. 
3.3. Proposition. A max-bounded frame is $T_{1}$-spatial if and only if it is subfit.

Proof. $\Leftarrow$ : Let $a \not \leq b$. Take a $c$ such that $a \vee c=1 \neq b \vee c$, and a maximal $p \geq b \vee c$. Then $p \nsupseteq a$ (else $p \geq a \vee c=1$ ) so that $a \not \leq p \geq b$.

$\Rightarrow$ : Let $a \not \leq b$. Choose a maximal $c$ such that $a \not \leq c \geq b$. Then $a \vee c>c$ and hence by maximality $a \vee c=1$, and $b \vee c=c \neq 1$.

3.3.1. Corollary. (Isbell's Spatiality Theorem) A compact subfit frame is $T_{1}$-spatial.

(By Zorn's Lemma a compact frame is obviously max-bounded.)

3.3.2. Note. The representation by maximal elements does not necessarily mean a representation by the whole of the spectrum. In such a representation of a $T_{1}$-space all of the maximal elements have to be present, but the spectrum can have more points (the remaining, non-maximal primes, would constitute together with the maximal ones the sobrification of the space).

This can easily happen even to a compact space. Consider an infinite set $X$ with the topology of complements of finite sets plus $\emptyset$. It is a compact $T_{1}$ (and hence subfit) space, but not a sober one. In the spectrum there is an extra point $\omega$ (corresponding to the void set which is in this $\Omega(X)$ a prime element) such that $\overline{\{\omega\}}=X$ (so that the sobrification is not $T_{1}$ !).

3.4. $T_{D}$-spatiality (see [4]). A frame $L$ is $T_{D}$-spatial if $L \cong \Omega(X)$ with a $T_{D}$-space $X$.

Write $a \sqsubset b$ for immediate precedence, that is, for the situation where $a<b$ and if $a \leq x \leq b$ then either $a=x$ or $x=b$. The following characteristic is in [4].

3.4.1. Proposition. A frame is $T_{D}$-spatial if and only if for any $a<b$ there are $u, v$ with $a \leq u \sqsubset v \leq b$.

3.5. A frame is step-bounded if for each $a<1$ there are $u, v$ such that

$$
a \leq u \sqsubset v .
$$

3.5.1. Lemma. A $T_{1}$-spatial frame satisfies the formula from 3.4.1.

Proof. Let $a<b$. Pick a maximal $p$ such that $a \leq p \nsupseteq b$. Thus, $a \leq b \wedge p<b$. Let $b \wedge p<x \leq b$. Then $x \not \leq p$ since otherwise $x \leq b \wedge p$; consequently $x \vee p \neq p$, and by maximality $x \vee p=1$. Thus,

$$
b=b \wedge(x \vee p)=(b \wedge x) \vee(b \wedge p)=x \vee(b \wedge p)=x
$$

so that $b \wedge p \sqsubset b$. 
3.5.2. Proposition. A subfit step-bounded frame is $T_{1}$-spatial.

Proof. For $a<1$ choose $u, v$ with $a \leq u \sqsubset v$. By subfitness there is a $c$ such that $v \vee c=1 \neq u \vee c$.

We will prove that $u \vee c$ is maximal. Consider an $x$ with $u \vee c<x$. We have

$$
u \leq x \wedge v \leq v
$$

so that either $u=x \wedge v$ or $v=x \wedge v$. If $u=x \wedge v$ then we have a contradiction

$$
x=x \wedge(v \vee c)=(x \wedge v) \vee(x \wedge c)=u \vee(x \wedge c)=(u \vee x) \wedge(u \vee c)<x .
$$

Hence $x \wedge v=v$. That is, $x \geq v$, and we see that $x \geq u \vee c \vee v=1$, and $u \vee c \geq a$ is maximal.

Thus, $L$ is max-bounded and using subfitness again we conclude by 3.3 that it is $T_{1}$-spatial.

3.6. Theorem. The following statements about a frame $L$ are equivalent:

(1) $L$ is $T_{1}$-spatial.

(2) $L$ is $T_{D}$-spatial and subfit.

(3) L is step-bounded and subfit.

Proof. (1) $\Rightarrow(2)$ follows from 3.3 and 3.5.1. Note that 3.3 is applicable because $L$, being $T_{1}$-spatial, is indeed max-bounded.

$(2) \Rightarrow(3)$ is trivial.

$(3) \Rightarrow(1)$ is in 3.5.2.

Now we can justify the definition in 3.2 .

3.6.1. Corollary. A frame is $T_{1}$-spatial if and only if it is isomorphic to an $\Omega(X)$ with a $T_{1}$-space $X$.

( $\Rightarrow$ is trivial. On the other hand, an $\Omega(X)$ with a $T_{1}$-space $X$ is obviously subfit and a $T_{D}$-frame.)

3.6.2. Note. The point is in the equivalence $(1) \equiv(3)$ which seems to be new (we have already mentioned above the standard fact that for a space, $T_{1}$ is equivalent to $T_{D} \&$ (subfit)).

3.7. Recall 3.3. Since a finite space is $T_{1}$ only if it is discrete we immediately obtain that

a finite distributive lattice is a Boolean algebra iff it is subfit.

This is a part of a much more general statement. The point is that the dual of a (finite) distributive lattice is again a (finite) distributive lattice and that, while the weak subfitness is a very weak condition indeed, its dual

$$
b<1 \Rightarrow \exists c, c \neq 0 \text {, and } b \wedge c=0
$$


that is,

$$
b^{*}=0 \Rightarrow b=1
$$

is in our context a very strong one.

3.7.1. Proposition. A pseudocomplemented distributive lattice (in particular, a frame) is a Boolean algebra if and only if it is dually weakly subfit.

Proof. Suppose the pseudocomplement $x^{*}$ of some $x \in L$ is not a complement, that is, $x \vee x^{*} \neq 1$. If we have (3.7.1) there is a $c \neq 0$ such that $c \wedge\left(x \vee x^{*}\right)=$ $(c \wedge x) \vee\left(c \wedge x^{*}\right)=0$, hence $c \wedge x=0$ so that $c \leq x^{*}$ and since also $c \wedge x^{*}=0$ we have a contradiction $c=0$.

3.7.2. Since a Boolean algebra is fit (indeed regular) we have

Corollary. For finite frames the subfitness is hereditary.

3.7.3. Note. Thus, a finite frame that is not subfit (i.e., a Boolean algebra) cannot be a sublocale of a finite subfit frame. But with infinite extensions the situation is different. Consider the following example.

In the set $\omega+1=\{0,1, \ldots, \omega\}$ take the topology consisting of the empty set and the complements of finite sets that contain $\omega$. The obtained space is easily seen to be subfit, but it contains (a.o.) the Sierpiński space

$$
(\{0, \omega\},\{\emptyset,\{\omega\},\{0, \omega\}\}) .
$$

\section{Replete subobjects}

4.1. We say that a subspace $Y$ of a space $X$ (more generally, a sublocale $S$ of a locale $L$ ) is replete if for each closed $A \neq \emptyset$ (each $a \neq 1$ ), $A \cap Y \neq \emptyset$ $(\mathfrak{c}(a) \cap S \neq 0$ ) (compare with the density where we meet the subspace with open subsets).

4.2. Recall that a frame homomorphism $h: L \rightarrow M$ is codense if $h(a)=1$ implies $a=1$.

4.2.1. Proposition. Let $f: M \rightarrow L$ be the right Galois adjoint of a frame homomorphism $h: L \rightarrow M$. Then $f[M]$ is replete in $L$ if and only if $h$ is codense.

Proof. We have

$$
\mathfrak{c}(a) \cap f[M]=\{f(x) \mid a \leq f(x)\}=\{f(x) \mid h(a) \leq x\} .
$$

Thus, $\mathfrak{c}(a) \cap f[M] \neq \mathrm{O}=\{1\}$ iff $f(h(a))<1$ and hence the repleteness of $f[M]$ amounts to the implication $a<1 \Rightarrow f(h(a))<1$, that is, $f(h(a))=$ $1 \Rightarrow a=1$. Since $1 \leq f(h(a))$ iff $1=h(1) \leq h(a)$ this is equivalent to $h(a)=1 \Rightarrow a=1$. 
4.3. The semiopen sublocales are defined by the formula

$$
\mathfrak{s o}(a)=\bigvee\{\mathfrak{c}(u) \mid \mathfrak{c}(u) \subseteq \mathfrak{o}(a)\} .
$$

(compare with the semiclosed sublocales

$$
\mathfrak{s c}(a)=\bigwedge\{\mathfrak{o}(u) \mid \mathfrak{c}(a) \subseteq \mathfrak{o}(u)\}
$$

from [14]). We obviously have

$$
\begin{aligned}
\mathfrak{s o}(a) & =\bigvee\{\mathfrak{c}(u) \mid \mathfrak{c}(u) \cap \mathfrak{c}(a)=\mathrm{O}\} \\
& =\bigvee\{\mathfrak{c}(u) \mid \mathfrak{c}(u \vee a)=\mathrm{O}\}=\bigvee\{\mathfrak{c}(u) \mid u \vee a=1\}
\end{aligned}
$$

4.4. Proposition. In every frame $L, \mathfrak{s o}(a) \wedge \mathfrak{c}(a)=\mathrm{O}$ and $\mathfrak{s o}(a) \vee \mathfrak{c}(a)$ is replete.

Proof. The first is trivial since $\mathfrak{s o}(a) \subseteq \mathfrak{o}(a)$. Now let $\mathfrak{c}(b) \neq \mathrm{O}$ (that is, $b \neq 1)$. If $\mathfrak{c}(b) \wedge \mathfrak{c}(a)=\mathrm{O}$ then $b \vee a=1$ and $\mathfrak{c}(b) \subseteq \mathfrak{s o}(a)$.

4.5. Proposition. The following statements about a frame $L$ are equivalent:

(1) $L$ is subfit.

(2) Every replete sublocale $S \subseteq L$ is equal to $L$.

(3) For every $a \in L, \mathfrak{s o}(a)=\mathfrak{o}(a)$.

Proof. (1) $\Rightarrow(2)$ : Let $S$ be replete and let $a \in L$. Set $s=\bigwedge\{t \mid t \in S, a \leq t\}$. Suppose $s \vee c=1$ and $a \vee c \leq t \in S$. Then $a \leq t$ and hence $s \leq t$, and since $c \leq t$ we have $1=s \vee c \leq t$. By repleteness, $a \vee c=1$, so that $s \vee c=1$ implies $a \vee c=1$ and by subfitness $s \leq a$ and $a=s \in S$.

$(2) \Rightarrow(3):$ By $4.4, \mathfrak{s o}(a) \wedge \mathfrak{c}(a)=\mathrm{O}$ and $\mathfrak{s o}(a) \vee \mathfrak{c}(a)=L$ so that $\mathfrak{s o}(a)=\mathfrak{o}(a)$, the unique complement.

$(3) \Rightarrow(1)$ : Let (3) hold and let $a \not \leq b$. Then $\mathfrak{c}(b) \nsubseteq \mathfrak{c}(a)$ and since $\mathfrak{c}(b)=$ $\mathfrak{c}(b) \cap(\mathfrak{s o}(a) \vee \mathfrak{c}(a))$ we have $\mathfrak{c}(b) \cap \mathfrak{s o}(a) \neq \mathrm{O}$ and hence for some $c$ with $c \vee a=1, \mathfrak{c}(b) \cap \mathfrak{c}(c)=\mathfrak{c}(b \vee c) \neq 0$, that is, $b \vee c \neq 1$.

4.6. Here is a related characteristic of weak subfitness $(\operatorname{int}(S)$ denotes the interior of a sublocale $S$, the largest open sublocale contained in $S$ ); slightly surprisingly, it comes in terms of semiclosed sublocales rather than in terms of semiopen ones.

Proposition. A frame $L$ is weakly subfit if and only if $\operatorname{int}(\mathfrak{s c}(a) \wedge \mathfrak{o}(a))=\mathrm{O}$ for every $a \in L$.

Proof. Assume $L$ is weakly subfit and let $\mathfrak{o}(b) \subseteq \mathfrak{s c}(a) \wedge \mathfrak{o}(a)$. Then $b \leq a$ and

$$
\mathfrak{o}(u) \geq \mathfrak{c}(a) \Rightarrow \mathfrak{o}(u) \geq \mathfrak{o}(b),
$$

that is,

$$
u \vee a=1 \Rightarrow u \geq b .
$$


Therefore, by weak subfitness, $b>0$ would imply the existence of a $u \neq 1$ such that $b \vee u=1$, which is absurd: by (*), from $1=b \vee u \leq a \vee u$ would follow $u \geq b$ and then $1=u \vee b=u$. Hence $b=0$.

Conversely, let $a>0$. Since $\operatorname{int}(\mathfrak{s c}(a) \wedge \mathfrak{o}(a))=\mathrm{O}$, we have in particular that $\mathfrak{o}(a) \nsubseteq \mathfrak{s c}(a)$. This means that there is some $u$ such that $\mathfrak{c}(a) \subseteq \mathfrak{o}(u)$ (i.e., $a \vee u=1)$ and $\mathfrak{o}(a) \nsubseteq \mathfrak{o}(u)$ (i.e., $a \not \leq u$ and so $u \neq 1$ ).

4.8. Repleteness in spaces. Recall the cong-equivalence $\approx$ from 2.4 .

4.8.1. Observation. If $Y \approx Z$ and $W$ is open then $W \cap Y \approx W \cap Z$. (Indeed, $U \cap(W \cap Y)=(U \cap W) \cap Y$ iff $(U \cap W) \cap Z=U \cap(W \cap Z)$.)

4.8.2. If $X$ is not $T_{D}$ we cannot infer the non-existence of a replete subset from subfitness. But we have at least the following

Proposition. Let $Y$ be replete in a subfit $X$. Then $Y \approx X$.

Proof. Suppose $U \cap Y=V \cap Y$ and $U \neq V$, say let there be an $x \in U \backslash V$. Take an $y \in \overline{\{x\}}$ such that $A=\overline{\{y\}} \subseteq U$. Since $V$ is open, $\overline{\{x\}} \cap V=\emptyset$ and hence also $A \cap V=\emptyset$ and $A \cap(V \cap Y)=\emptyset$. On the other hand, $A \cap U \cap Y=A \cap Y \neq \emptyset$, by repleteness.

The converse holds in every space:

Proposition. Let $Y$ be a subspace of a space $X$ such that $Y \approx X$. Then $Y$ is replete.

Proof. $Y \approx X$ means that

$$
A \cap Y=B \cap Y \text { iff } A=B \text { for every open } A, B .
$$

In particular, for $B=X$, we have

$$
Y \subseteq A \text { iff } A=X \text { for every open } A .
$$

Now suppose $Y$ is not replete. Then there is some closed $F \neq \emptyset$ such that $Y \cap F=\emptyset$. Therefore $X \backslash F$ is an open set containing $Y$. Hence $F=\emptyset$, a contradiction.

4.9. For any subspace $Y \subseteq X$ (not only for an open one) set

$$
\mathfrak{s}(Y)=\bigcup\{A \mid A \text { closed, } A \subseteq Y\} .
$$

We have

4.9.1. Lemma. $\mathfrak{s}(Y) \cup(X \backslash Y)$ is replete.

Proof. Let $C$ be non-void closed. Consider $\mathfrak{s}(Y) \cup(X \backslash Y) \cap C$. If $C \cap(X \backslash Y)=$ $\emptyset$ then $C \subseteq Y$ and hence $C \subseteq \mathfrak{s}(Y)$. 
Now the cong-equivalence relation yields a new characteristic of subfit spaces.

4.9.2. Proposition. A space is subfit if and only if $U \approx \mathfrak{s}(U)$ for every open $U$.

Proof. The implication " $\Rightarrow$ " follows from 4.9.1 and 4.8.1. For the converse implication use the Isbell-Simmons Theorem (see 2.3). Let $U$ be an open set, $x \in U$. The condition $U \approx \mathfrak{s}(U)$ means that

$$
A \cap U=B \cap U \text { iff } A \cap \mathfrak{s}(U)=B \cap \mathfrak{s}(U) \text { for every open } A, B .
$$

In particular, for $B=U$ we have

$$
U \subseteq A \quad \text { iff } \quad \mathfrak{s}(U) \subseteq A \text { for every open } A .
$$

Applying $(*)$ to $U \nsubseteq X \backslash \overline{\{x\}}$ we conclude that $\mathfrak{s}(U) \nsubseteq X \backslash \overline{\{x\}}$, that is, $\mathfrak{s}(U) \cap \overline{\{x\}} \neq \emptyset$. Hence there is a $y \in \overline{\{x\}}$ and a closed $F \subseteq U$ containing $y$.

\section{MEET FORMULAS FOR PSEUdOCOMPLEMENT \\ AND HEYTING OPERATION}

5.1. There is a surprising formula for pseudocomplement as the meet $a^{*}=$ $\bigwedge\{x \mid a \vee x=1\}$, valid under suitable circumstances. In the literature it was first encountered as a fact about zero-dimensional frames, but it must be a folklore that it holds more generally (for instance there is a very natural proof by computation in [2] that this formula, and a more general one for the Heyting operation, holds for regular frames). In his recent note [10], concerned more generally with d-frames, Olaf Klinke observed that in fact the pseudocomplement formula holds already for weakly subfit frames (he claimed fitness, but when analysing his proof the week subfitness emerges). In fact we will see that the formulas lead to characteristics of subfitness and weak subfitness as in 5.2 below.

5.2. Theorem. In a frame $L$, the formula

$$
a \rightarrow b=\bigwedge\{x \mid a \vee x=1, x \geq b\}
$$

for the Heyting operation is valid if and only if $L$ is subfit.

The formula

$$
a^{*}=\bigwedge\{x \mid a \vee x=1\}
$$

for pseudocomplement is valid if and only if $L$ is weakly subfit.

(Thus in particular in weakly subfit frames that are not subfit we have the "almost supplement" formula (5.2.2) for pseudocomplement, but not the meet formula (5.2.1) for the Heyting operation.) 
Proof. I. Set $u=\bigwedge\{x \mid a \vee x=1\}$. If $a \vee x=1$ then $a^{*}=a^{*} \wedge(a \vee x)=a^{*} \wedge x$; hence $a^{*} \leq u$.

Suppose $a \wedge u \neq 0$. Hence there is an $x \neq 1$ such that $(a \wedge u) \vee x=$ $(a \vee x) \wedge(u \vee x)=1$ and hence $a \vee x=u \vee x=1$. Then, however, by the formula for $u$ and the first equality we have $u \leq x$, and the second equality yields $x=1$, a contradiction.

II. Now let $L$ be subfit. Note that

- for $x \geq b$, the element $x \rightarrow b$ is the pseudocomplement $x^{* b}$ of $x$ in the closed sublocale $\uparrow b$, and

- $\uparrow b$ is closed under meets and non-empty joins.

Hence we have

$$
\begin{aligned}
a \rightarrow b & =(a \rightarrow b) \wedge(b \rightarrow b)=(a \vee b) \rightarrow b=(a \vee b)^{* b}= \\
& =\bigwedge\{x \mid a \vee b \vee x=1, x \geq b\}=\bigwedge\{x \mid a \vee x=1, x \geq b\} .
\end{aligned}
$$

III. Let $L$ not be weakly subfit. Then there is an $a \in L$ such that $a>0$ and $a \vee x=1$ implies $x=1$. Then $a^{*} \neq 1$ while $\bigwedge\{x \mid a \vee x=1\}=1$.

IV. Let $L$ not be subfit Then there is a $b \in L$ such that $\uparrow b$ is not weakly subfit and hence there is by III also an $a>b$ such that $a \rightarrow b=a^{* b} \neq \bigwedge\{x \mid a \vee x=$ $1, x \geq b\}$.

5.3. Note. The following fact goes back to Isbell. An element is linear if it join-distributes over arbitrary meets.

Fact. An element a of a subfit frame is complemented iff it is linear.

Note that this is, already for weakly subfit frames, an immediate consequence of 5.2.

5.4. Note. Recall the concept of prefitness from [14],

$$
\forall a>0 \exists c, c^{*} \neq 0 \text { and } a \vee c=1 .
$$

Obviously (prefit) implies (weakly subfit), and these two concepts look formally very close $\left(c^{*} \neq 0\right.$ replaces $\left.c \neq 1\right)$. In actual fact, however, they are worlds apart.

Let us look closer at the situation. Weak subfitness is weaker than subfitness and this is still weaker than $T_{1}$ in spaces. On the other hand, prefitness is already close to regularity: a frame $L$ is prefit iff for each $a \in L$,

$$
a \leq(\bigvee\{x \mid x \prec a\})^{* *}
$$

(hence, it is "regular up to density"), see [14].

Somewhat surprisingly, prefitness does not imply subfitness. Thus, there exist frames that are "almost regular", in which the formula (5.2.2) holds while the (5.2.1) does not! 


\section{REFERENCES}

[1] C. E. Aull and W. J. Thron, Separation axioms between $T_{0}$ and $T_{1}$, Indag. Math. 24 (1963) 26-37.

[2] R. N. Ball, J. Picado and A. Pultr, On an aspect of scatteredness in the pointfree setting, Port. Math. 73 (2016) 139-152.

[3] B. Banaschewski and A. Pultr, Variants of openness, Appl. Categ. Structures 2 (1994) 331-350.

[4] B. Banaschewski and A. Pultr, Pointfree aspects of the $T_{D}$ axiom of classical topology, Quaest. Math. 33 (2010) 369-385.

[5] C. H. Dowker and D. Strauss, $T_{1}$ - and $T_{2}$-axioms for frames, in: Aspects of topology, London Math. Soc. Lecture Note Ser. 93, pp. 325-335, Cambridge Univ. Press, Cambridge, 1985 .

[6] H. Herrlich, A concept of nearness, Gen. Topology Appl. 5 (1974) 191-212.

[7] H. Herrlich and A. Pultr, Nearness, subfitness and sequential regularity, Appl. Categ. Structures 8 (2000) 67-80

[8] J. R. Isbell, Atomless parts of spaces, Math. Scand. 31 (1972) 5-32.

[9] P. T. Johnstone, Stone Spaces, Cambridge Univ. Press, Cambridge, 1982.

[10] O. Klinke, When consistency is redundant, manuscript, April 2015.

[11] K. Morita, On the simple extension of a space with respect to a uniformity I, Proc. Japan Acad. 27 (1951) 65-72.

[12] J. Picado and A. Pultr, Frames and Locales: topology without points, Frontiers in Mathematics, Vol. 28, Springer, Basel, 2012.

[13] J. Picado and A. Pultr, (Sub)Fit biframes and non-symmetric nearness, Topology Appl. 168 (2014) 66-81.

[14] J. Picado and A. Pultr, More on subfitness and fitness, Appl. Categ. Structures 23 (2015) 323-335.

[15] A. Pultr, Frames, in: Handbook of Algebra (ed. by M. Hazewinkel), vol. 3, pp. 791-858, Elsevier, 2003.

[16] H. Simmons, The lattice theoretic part of topological separation properties, Proc. Edinburgh Math. Soc. (2) 21 (1978) 41-48.

[17] H. Simmons, Regularity, fitness, and the block structure of frames, Appl. Categ. Structures 14 (2006) 1-34.

CmuC, Department of Mathematics, University of Coimbra, Apartado 3008, 3001-501 Coimbra, Portugal

E-mail address: picado@mat.uc.pt

Department of Applied Mathematics and iti, MFF, Charles University, Malostranské nám. 24, 11800 Praha 1, Czech Republic

E-mail address: pultr@kam.ms.mff.cuni.cz 